\title{
A novel approach of the conformal mappings with applications in biotribology
}

\author{
Olivia Florea
}

\begin{abstract}
In this paper, the flow of an incompressible non Newtonian fluid between two eccentric cylinders is considered. The aim of this study is to determine the flow in the case of the stationary movement of some viscous fluids between two eccentric cylinders with the generators parallel with $\mathrm{Oz}$ axis. Using the Mobius conformal mapping are obtained two concentric cylinders. The expression of velocity is deduced with the separation of variable method.
\end{abstract}

\section{Indroduction}

The flow of fluid through a ring is a classical problem that had attracted several researchers because of its enormous applications in the real life. An analytic solution for the flow of viscous Newtonian fluid through vertical ring can be found in the classical textbooks of Bird. et.al [3].

Studies [7]-[18] present the analytic and numerical results for the flow of different type of fluids between concentric cylinders. In this paper we will consider the case when the geometry of the two cylinders is not concentric. The aim of this study is to determine the flow in the case of the stationary movement of some viscous fluids between two non-concentric cylinders with the generators parallel with $O z$ axis. It is considered that the viscous fluid

Key Words: Navier-Stokes equation, pressure gradient, conformal mapping.

2010 Mathematics Subject Classification: Primary 34M40,35K50, 35Q30; Secondary $76 \mathrm{D} 05$.

Received: 30 April, 2014.

Revised: 20 May, 2014.

Accepted: 30 June, 2014. 
is incompressible and the section of the movement domain is the ring delimited by two circles with the centers on $O x$ axis: $\left(\mathfrak{C}_{2}, O, r_{2}\right),\left(\mathfrak{C}_{1}, O_{1}, r_{1}\right)$, with $r_{1}<r_{2}$, the circle $\mathcal{C}_{1}$ is inside of the circle $\mathcal{C}_{2}$, the distance between the two centers of the circles is $O O_{1}=d$.

We have to solve the problem of the movement of the viscous fluid which is generated by the pressure gradient $\frac{\partial p}{\partial z}=k_{1}$ and by the gravitational potential $-\rho g \sin (\alpha)$, the angle between the generators and the $O x$ axis is $\alpha$. To solve the proposed problem it is used the conformal mapping method and the variable separation method.

The problem of reflection and transmission of plane waves at an imperfect boundary between two thermally conducting micropolar elastic solid half spaces with two temperature is investigated in the papers [16], [11]. For the boundary value problem considered in the context of dipolar bodies with stretch, in the paper [12],[10] the authors use some results from the theory of semigroups of the linear operators in order to prove the existence and uniqueness of a weak solution. In the papers [13], [14] the authors have studied different types of problems in microstretch thermoelastic medium.

This study has multiple applications in biotribology and lubrification, in the thermodynamics of viscous fluids. The mathematical model can be applied in the study of the torsion of elastic fibers, in thermoelasticity or electromagnetism. In the study of rheology, rheometry is used to experimentally determine rheological properties of materials. A rheometer is an instrument, which can impose a strain and measures the resulting torque or it can exert a torque on a material and measures its response with time. A rheometer can be of the controlled stress type or the controlled rate type. To characterize the rheological behavior of the material, different flow test techniques such as steady shear or oscillatory shear could be used. The measuring systems used on the rheometer can be selected from the following geometries based on the material properties: cone and plate, parallel plate, concentric cylinder [2], [5]. A fluid sample is introduced between the inner and outer cylindrical surfaces, which are disposed eccentrically relative to one another. Relative mechanical movement of the members, which is related to both shear and displacement of the liquid disposed between them, provides an indication of the rheological properties of the liquid, [9], [17]. A new approach of a non newtonian fluid in the knee osteoarthrosis for the synovial fluid was presentetd in [6], where the study is about the Stokes' second problem, when the wall is driven in an oscillatory shearing motion. 


\section{Formulation of the problem}

The equations of movement Navier - Stokes for the incompressible, viscous fluids on which acts the gravitational force are [1]:

$$
\left\{\begin{array}{l}
\frac{d u}{d t}=-\frac{1}{\rho} \frac{\partial p}{\partial x}+\nu \cdot \nabla^{2} u \\
\frac{d v}{d t}=-\frac{1}{\rho} \frac{\partial p}{\partial y}+\nu \cdot \nabla^{2} v \\
\frac{d w}{d t}=-\frac{1}{\rho}\left(\frac{\partial p}{\partial z}-\rho g \sin (\alpha)\right)+\nu \cdot \nabla^{2} w
\end{array}\right.
$$

where

$$
\begin{gathered}
\frac{d}{d t}=\frac{\partial}{\partial t}+u \frac{\partial}{\partial x}+v \frac{\partial}{\partial y}+w \frac{\partial}{\partial z} \\
\nabla^{2}=\frac{\partial^{2}}{\partial x^{2}}+\frac{\partial^{2}}{\partial y^{2}}+\frac{\partial^{2}}{\partial z^{2}}
\end{gathered}
$$

here, $p$ is the pressure, $\rho$ is the density of the fluid, $\mu$ is the kinematic viscosity coefficient. The equation of continuity is:

$$
\frac{\partial u}{\partial x}+\frac{\partial v}{\partial y}+\frac{\partial w}{\partial z}=0
$$

if the flow direction is supossed to be parallel with $O z$ axis, then the velocity has the direction of $z$ if $u$ and $v$ vanish. Therefore, the continuity equation became:

$$
\frac{\partial w}{\partial z}=0 \quad \text { or } \quad w=w(x, y, t)
$$

this relation shows that the velocity is constant on a parallel direction with the central line. By substituting $u=v=0$ we have: $\frac{\partial p}{\partial x}=0 ; \frac{\partial p}{\partial y}=0$. Based on the mentioned considerations the third equation form (1) can be written in the following form:

$$
\frac{d w}{d t}=\frac{\partial w}{\partial t}+u \frac{\partial w}{\partial x}+v \frac{\partial w}{\partial y}+w \frac{\partial w}{\partial z} \Leftrightarrow \frac{d w}{d t}=\frac{\partial w}{\partial t}
$$

or

$$
\frac{\partial w}{\partial t}=-\frac{1}{\rho}\left(\frac{\partial p}{\partial z}-\rho g \sin (\alpha)\right)+\nu\left(\frac{\partial^{2} w}{\partial x^{2}}+\frac{\partial^{2} w}{\partial y^{2}}\right)
$$

Writing the above equation in polar coordinates we have:

$$
\frac{\partial w}{\partial t}=-\frac{1}{\rho}\left(\frac{\partial p}{\partial z}-\rho g \sin (\alpha)\right)+\nu\left(\frac{\partial^{2} w}{\partial r^{2}}+\frac{1}{r} \frac{\partial w}{\partial r}+\frac{1}{r^{2}} \frac{\partial^{2} w}{\partial \theta^{2}}\right)
$$


The initial boundary conditions are:

$$
\left\{\begin{array}{l}
w(r, \theta, t=0)=0 \\
\left.w(r, \theta, t)\right|_{\mathcal{e}_{1}}=\left.w(r, \theta, t)\right|_{\mathcal{C}_{2}}=0
\end{array}\right.
$$

The fluid flow is assured by the gravitational effect; we divide by $\nu$, the dynamic viscosity is $\mu=\nu \rho$, and it is obtained:

$$
\frac{1}{\mu} \frac{\partial w}{\partial t}=-\frac{1}{\nu \rho}\left(\frac{\partial p}{\partial z}-\rho g \sin \alpha\right)+\frac{\partial^{2} w}{\partial r^{2}}+\frac{1}{r} \frac{\partial w}{\partial r}+\frac{1}{r^{2}} \frac{\partial^{2} w}{\partial \theta^{2}}
$$

Noting by $\Omega=\frac{\partial p}{\partial z}-\rho g \sin \alpha$ and neglecting the derivative of $w$ in report with the time, the equation above becomes:

$$
\frac{\partial^{2} w}{\partial r^{2}}+\frac{1}{r} \frac{\partial w}{\partial r}+\frac{1}{r^{2}} \frac{\partial^{2} w}{\partial \theta^{2}}=\frac{\Omega}{\mu}
$$

that is equivalent with

$$
\Delta=\frac{\Omega}{\mu}
$$

Lemma 1. The equation (6) has the particular solution:

$$
w_{p}=\frac{\Omega}{2 \mu} r^{2} \sin ^{2} \theta
$$

Proof.

$$
\begin{array}{cl}
\frac{\partial w_{p}}{\partial r}=\frac{\Omega r}{\mu} \sin ^{2} \theta, & \frac{\partial^{2} w_{p}}{\partial r^{2}}=\frac{\Omega}{\mu} \sin ^{2} \theta, \\
\frac{\partial w_{p}}{\partial \theta}=\frac{\Omega r^{2}}{2 \mu} \sin (2 \theta), & \frac{\partial^{2} w_{p}}{\partial \theta^{2}}=\frac{\Omega r^{2}}{\mu} \cos (2 \theta)
\end{array}
$$

Replacing in (6) it is obtained the identity:

$$
\frac{2 \Omega}{2 \mu} \sin ^{2} \theta+\frac{\Omega}{\mu}\left(1-2 \sin ^{2} \theta\right)=\frac{\Omega}{\mu} \Leftrightarrow \frac{\Omega}{\mu}=\frac{\Omega}{\mu}
$$

In the equation (6) we perform the change of unknown function $W=w-w_{p}$ which will lead to the homogeneous equation:

$$
\Delta W=0
$$




\section{Main results}

To solve the proposed problem it is absolutely necessary for the two cylinders to be concentric with the center in the origin of coordinates system. It is necessary a conformal mapping of Möbius type such as the new circles will be: $C_{1}^{\prime}\left(0, \frac{1}{a}\right), C_{2}^{\prime}(0, a)$. In the fig. 1 are represented the transversal sections for the geometry of the cylinders before and after the conformal mapping:
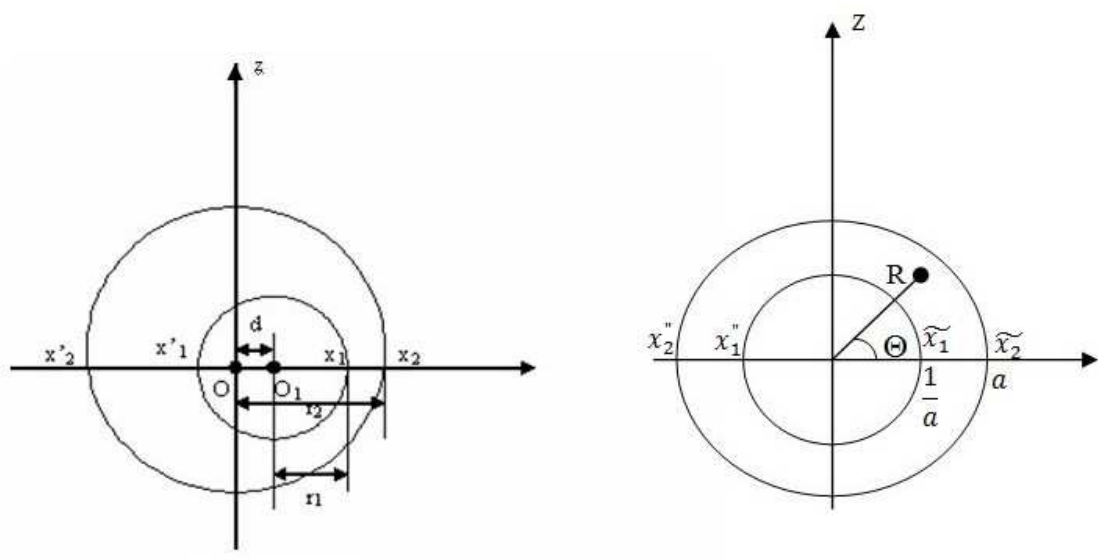

Figure 1: (a) initial geometry of eccentric cylinders; (b) the Mobius conformal mapping

Lemma 2. The conformal mapping that transforms the circles $\mathcal{C}_{1}\left(O_{1}(d, 0), r_{1}\right), \mathcal{C}_{2}\left(O(0,0), r_{2}\right)$ into the circles $C_{1}^{\prime}\left(0, \frac{1}{a}\right), C_{2}^{\prime}(0, a)$ is:

$$
Z=a \frac{z-b r_{2}}{r_{2}-b z}=f(z), b \in(0,1)
$$

Proof. We verify that the conformal mapping is well chosen:

for $z=r_{2}$ we have: $f\left(r_{2}\right)=a$ and for $z=-r_{2}$ we have: $f\left(-r_{2}\right)=-a$.

For the homographic transformation to exist $Z=\frac{a z+b}{c z+d}$ the complex coefficients $a, b, c, d$ satisfy the condition $a d-b c \neq 0$. In our case the complex coefficients satisfy the condition $r_{2}-b^{2} r_{2}=r_{2}\left(1-b^{2}\right)>0,(\forall) b \in(0,1)$.

The homographic transform (9) impose the following conversions:

$$
f\left(x_{1}^{\prime}\right) \rightarrow x_{1}^{\prime \prime} ; \quad f\left(x_{1}\right) \rightarrow \tilde{x}_{1}
$$


where $\tilde{x}_{1}=-x_{1}^{\prime \prime}$ and $x_{1}=r_{1}+d ; x_{1}^{\prime}=d-r_{1}$. Replacing the previous notations we have:

$$
f\left(x_{1}^{\prime}\right)=-\frac{1}{a} ; f\left(x_{1}\right)=\frac{1}{a} .
$$

These are equivalent with $f\left(x_{1}\right)=-f\left(x_{1}^{\prime}\right)$ and in this manner we will obtain an equation in the unknown $b$ :

$$
\frac{r_{1}+d-b r_{2}}{r_{2}-b r_{1}-b d}=-\frac{d-r_{1}-b r_{2}}{r_{2}-b d+b r_{1}}
$$

that is equivalent with:

$$
\frac{\frac{r_{1}+d}{r_{2}}-b}{1-b \frac{r_{1}+d}{r_{2}}}=-\frac{\frac{r_{1}-d}{r_{2}}-b}{1-b \frac{d-r_{1}}{r_{2}}}
$$

To simplify the equation (10) we make the following notations:

$$
\frac{r_{1}+d}{r_{2}}=p ; \quad \frac{d-r_{1}}{r_{2}}=q ; \quad 0<q<p<1 .
$$

Replacing these new notations in (10) it is obtained an algebraic equation of second degree:

$$
b^{2}(p-q)+2 b(p q-1)+p-q=0
$$

with the solutions:

$$
b_{1,2}=\frac{1-p q \pm \sqrt{\left(p^{2}-1\right)\left(q^{2}-1\right)}}{p-q}
$$

Because it is necessary to respect the imposed condition, that $b \in(0,1)$ the convenient solution is:

$$
b=\frac{1-p q-\sqrt{\left(p^{2}-1\right)\left(q^{2}-1\right)}}{p-q}
$$

Next we have to determine the value of parameter $a>0$, and for this we will use the relation:

$$
f\left(x_{1}\right)=\frac{1}{a} \Leftrightarrow a^{2}=\frac{r_{2}-b\left(r_{1}+d\right)}{r_{1}+d-b r_{2}} \Leftrightarrow a^{2}=\frac{1-b p}{p-b}
$$

Solving the last relation we obtain:

$$
a=\sqrt{\frac{p^{2} q-q+p \sqrt{\left(p^{2}-1\right)\left(q^{2}-1\right)}}{p^{2}-1+\sqrt{\left(p^{2}-1\right)\left(q^{2}-1\right)}}}
$$


The validation of the good choise of the conformal mapping is obtained with Maple software, see fig.2.

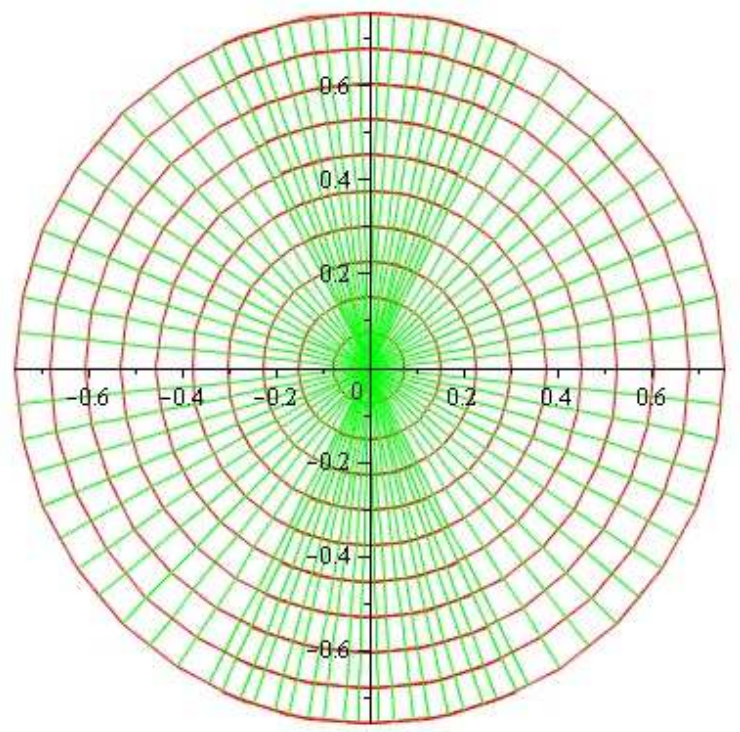

Figure 2: The conformal mapping

For the particular solution (7) we should apply the conformal mapping i.e.:

$$
y^{2}=r^{2} \sin ^{2} \theta=F(R, \Theta)
$$

From the relation (9) we can obtain the expression $z=F(Z)$ :

$$
z=r_{2} \frac{Z+a b}{a+b Z}
$$

using the algebric form of a complex number, relation (13) becomes:

$$
x+i y=r_{2} \frac{X+i Y+a b}{a+b(X+i Y)}
$$

Writing the conjugate of the above equation, we have:

$$
x-i y=r_{2} \frac{X-i Y+a b}{a+b(X-i Y)}
$$


Subtracting the two above equations it is obtained:

$$
y=r_{2} \frac{a Y\left(1-b^{2}\right)}{(a+b X)^{2}+m^{2} Y^{2}} \Leftrightarrow .
$$

The polar coordinates are:

$$
\left\{\begin{array}{l}
X=R \cos \Theta \\
Y=R \sin \Theta
\end{array} \Leftrightarrow R^{2}=X^{2}+Y^{2}\right.
$$

Replacing the polar coordinates in the equation (14) and then lifting at square we can write:

$y^{2}=r_{2}^{2} \frac{a^{2} Y^{2}\left(1-b^{2}\right)^{2}}{\left(a^{2}+2 a b X+b^{2} R^{2}\right)^{2}} \Leftrightarrow y^{2}=r_{2}^{2} \frac{a^{2} R^{2} \sin ^{2} \Theta\left(1-b^{2}\right)^{2}}{\left(a^{2}+2 a b R \cos \Theta+b^{2} R^{2}\right)^{2}}=F(R, \Theta)$

Based on the variable change $W=w-w_{p}$ the conditions on the boundary (5) become:

$$
\left\{\begin{array}{l}
\left.W\right|_{C_{1}}=\left.w\right|_{C_{1}}-\left.w_{p}\right|_{C_{1}}=0-\left.\frac{\Omega}{2 \mu} y^{2}\right|_{C_{1}}=-\frac{\Omega}{2 \mu} F\left(\frac{1}{a}, \Theta\right) \\
\left.W\right|_{C_{2}}=\left.w\right|_{C_{2}}-\left.w_{p}\right|_{C_{2}}=0-\left.\frac{\Omega}{2 \mu} y^{2}\right|_{C_{2}}=-\frac{\Omega}{2 \mu} F(a, \Theta)
\end{array}\right.
$$

Using the relations (14) the boundary conditions are:

$$
\left\{\begin{array}{l}
\left.W\right|_{C_{1}}=-\frac{\Omega}{2 \mu} \frac{r_{2}^{2}\left(1-b^{2}\right)^{2}}{a^{4}} \frac{\sin ^{2} \Theta}{\left(1+\frac{2 b}{a^{2}} \cos \Theta+\frac{b^{2}}{a^{4}}\right)^{2}} \\
\left.W\right|_{C_{2}}=-\frac{\Omega}{2 \mu} r_{2}^{2}\left(1-b^{2}\right)^{2} \frac{\sin ^{2} \Theta}{\left(1+2 b \cos \Theta+b^{2}\right)^{2}}
\end{array}\right.
$$

Noting by:

$$
\phi(\Theta)=\frac{\sin ^{2} \Theta}{\left(1+\frac{2 b}{a^{2}} \cos \Theta+\frac{b^{2}}{a^{4}}\right)^{2}} ; \quad \psi(\Theta)=\frac{\sin ^{2} \Theta}{\left(1+2 b \cos \Theta+b^{2}\right)^{2}}
$$

The boundary conditions can be written in the following form:

$$
\left\{\begin{array}{l}
\left.W\right|_{C_{1}}=-\frac{\Omega}{2 \mu} \frac{r_{2}^{2}\left(1-b^{2}\right)^{2}}{a^{4}} \phi(\Theta) \\
\left.W\right|_{C_{2}}=-\frac{\Omega}{2 \mu} r_{2}^{2}\left(1-b^{2}\right)^{2} \psi(\Theta)
\end{array}\right.
$$

Proposition 1. The particular solution for the equation (8) written in polar coordinates:

$$
\frac{\partial^{2} W}{\partial R^{2}}+\frac{1}{R} \frac{\partial W}{\partial R}+\frac{1}{R^{2}} \frac{\partial^{2} W}{\partial \Theta^{2}}=0
$$

is:

$$
W_{p}=A \ln R+B, \quad A, B \in \mathbf{R}
$$


Theorem 1. The general solution of the equation (18) is:

$$
W=A \ln R+B+\sum_{n=1}^{\infty}\left(a_{n} R^{n}+b_{n} R^{-n}\right) \cos (n \Theta),
$$

where $a_{n}$ and $b_{n}$ are the coefficients of Fourier series.

Proof. Using the variable separation method we want to find $W=X(R)$. $Y(\Theta)$. Replacing in (18) we have:

$$
X^{\prime \prime} Y+\frac{1}{R} X^{\prime} Y+\frac{1}{R^{2}} X Y^{\prime \prime}=0
$$

Dividing the above relation through $X Y$ we have:

$$
R^{2} \frac{X^{\prime \prime}}{X}+R \frac{X^{\prime}}{X}=-\frac{Y^{\prime \prime}}{Y}=\lambda^{2}
$$

Therefore, we will obtain two differential equations of second degree. First of them is:

$$
Y^{\prime \prime}+\lambda^{2} Y=0
$$

which has the solution $Y=C_{1} \cos (\lambda \Theta)$. Due to the fact that $W$ is an even function, $W(-\Theta)=W(\Theta)$ the sine term doesn't appear anymore. The second equation that is obtained from (21) is a differential equation of second degree of Euler type:

$$
R^{2} X^{\prime \prime}+R X^{\prime}-\lambda^{2} X=0
$$

with the solution $\tilde{X}=R^{n}$. Replacing this solution in Euler differential equation we obtain:

$$
n(n-1) R^{n}+n R^{n}-\lambda^{2} R^{n}=0 \Leftrightarrow \lambda= \pm n
$$

The solution of the Euler differential equation is:

$$
X(R)=C_{3} R^{\lambda}+C_{4} R^{-\lambda}
$$

Therefore, we have:

$$
W_{n}(R, \Theta)=X(R) \cdot Y(\Theta)=\left(a_{n} R^{n}+b_{n} R^{-n}\right) C_{1} \cos (n \Theta)
$$

this relation leads us to the solution of homogeneous equation:

$$
W_{o}(R, \Theta)=\sum_{n=1}^{\infty} W_{n}(R, \Theta)
$$


Hence, the general solution (20) is obtained. Returning to the boundary conditions (17) we have:

$$
\left\{\begin{array}{l}
\left.W\right|_{C_{1}}=-\frac{\Omega}{2 \mu} \frac{r_{2}^{2}\left(1-b^{2}\right)^{2}}{a^{4}} \phi(\Theta)=A \ln \frac{1}{a}+B+\sum_{n=1}^{\infty}\left(a_{n}\left(\frac{1}{a}\right)^{n}+b_{n}\left(\frac{1}{a}\right)^{-n}\right) \cos (n \Theta) \\
\left.W\right|_{C_{2}}=-\frac{\Omega}{2 \mu} r_{2}^{2}\left(1-b^{2}\right)^{2} \psi(\Theta)=A \ln a+B+\sum_{n=1}^{\infty}\left(a_{n} \cdot a^{n}+b_{n} \cdot a^{-n}\right) \cos (n \Theta)
\end{array}\right.
$$

We will use the Fourier method to determine the Fourier coefficients:

$$
\begin{aligned}
& -A \ln a+B=\frac{2}{\pi} \int_{0}^{\pi}-\frac{\Omega}{2 \mu} \frac{r^{2}\left(1-b^{2}\right)^{2}}{a^{4}} \phi(\Theta) d \Theta \\
& A \ln a+B=\frac{2}{\pi} \int_{0}^{\pi}-\frac{\Omega}{2 \mu} r_{2}^{2}\left(1-b^{2}\right)^{2} \psi(\Theta) d \Theta \\
& a_{n} \cdot a^{-n}+b_{n} \cdot a^{n}=\frac{2}{\pi} \int_{0}^{\pi}-\frac{\Omega}{2 \mu} \frac{r_{2}^{2}\left(1-b^{2}\right)^{2}}{a^{4}} \phi(\Theta) \cos (n \Theta) d \Theta \\
& a_{n} \cdot a^{n}+b_{n} \cdot a^{-n}=\frac{2}{\pi} \int_{0}^{\pi}-\frac{\Omega}{2 \mu} r_{2}^{2}\left(1-b^{2}\right)^{2} \psi(\Theta) \cos (n \Theta) d \Theta
\end{aligned}
$$

The Fourier development of the functions:

$$
\begin{aligned}
& \Phi(\Theta)=-\frac{\Omega}{2 \mu} \frac{r_{2}^{2}\left(1-b^{2}\right)^{2}}{a^{4}} \phi(\Theta) \\
& \Psi(\Theta)=-\frac{\Omega}{2 \mu} r_{2}^{2}\left(1-b^{2}\right)^{2} \psi(\Theta)
\end{aligned}
$$

in cosine series is:

$$
\begin{aligned}
& \Phi(\Theta)=\alpha_{0}^{1}+\sum_{n=1}^{\infty} \alpha_{n}^{1} \cos (n \Theta), \quad \text { for } \quad R=\frac{1}{a} \\
& \Psi(\Theta)=\alpha_{0}^{2}+\sum_{n=1}^{\infty} \alpha_{n}^{2} \cos (n \Theta), \quad \text { for } \quad R=a
\end{aligned}
$$

with:

$$
\begin{array}{ll}
\alpha_{0}^{1}=\frac{2}{\pi} \int_{0}^{\pi} \Phi(\Theta) d \Theta & \alpha_{0}^{2}=\frac{2}{\pi} \int_{0}^{\pi} \Psi(\Theta) d \Theta \\
\alpha_{n}^{1}=\frac{2}{\pi} \int_{0}^{\pi} \Phi(\Theta) \cos (n \Theta) d \Theta & \alpha_{n}^{2}=\frac{2}{\pi} \int_{0}^{\pi} \Psi(\Theta) \cos (n \Theta) d \Theta
\end{array}
$$

Based on the relations (25), the equations (23) can be written:

$$
\left\{\begin{array}{r}
-A \ln a+B=\alpha_{0}^{1} \\
A \ln a+B=\alpha_{0}^{2}
\end{array}\right.
$$


respectively,

$$
\left\{\begin{array}{l}
a_{n} \cdot a^{-n}+b_{n} \cdot a^{n}=\alpha_{n}^{1} \\
a_{n} \cdot a^{n}+b_{n} \cdot a^{-n}=\alpha_{n}^{1}
\end{array}\right.
$$

Hence, the coefficients from (23) function of $\alpha_{0}^{i}, \alpha_{n}^{i}, i=\overline{1,2}$ are:

$$
\begin{array}{lrl}
A=\frac{\alpha_{0}^{2}-\alpha_{0}^{1}}{2 \ln a} & B=\frac{\alpha_{0}^{1}+\alpha_{0}^{2}}{2} \\
a_{n}=\frac{\alpha_{n}^{2} a^{n}-\alpha_{n}^{1} a^{-n}}{a^{2 n}-a^{-2 n}} & b_{n}=\frac{\alpha_{n}^{1} a^{n}-\alpha_{n}^{2} a^{-n}}{a^{2 n}-a^{-2 n}}
\end{array}
$$

Next we determine the Fourier coefficients from (25). For this we have to compute the following integrals:

$$
\begin{aligned}
& \mathcal{J}_{0}^{1}=\frac{2}{\pi} \int_{0}^{\pi} \phi(\Theta) d \Theta=\frac{2}{\pi} \int_{0}^{\pi} \frac{\sin ^{2} \Theta}{\left(1+\frac{2 b}{a^{2}} \cos \Theta+\frac{b^{2}}{a^{4}}\right)^{2}} d \Theta \\
& \mathcal{J}_{0}^{2}=\frac{2}{\pi} \int_{0}^{\pi} \psi(\Theta) d \Theta=\frac{2}{\pi} \int_{0}^{\pi} \frac{\sin ^{2} \Theta}{\left(1+2 b \cos \Theta+b^{2}\right)^{2}} d \Theta \\
& \mathcal{J}_{n}^{1}=\frac{2}{\pi} \int_{0}^{\pi} \phi(\Theta) \cos (n \Theta) d \Theta=\frac{2}{\pi} \int_{0}^{\pi} \frac{\sin ^{2} \Theta}{\left(1+\frac{2 b}{a^{2}} \cos \Theta+\frac{b^{2}}{a^{4}}\right)^{2}} \cos (n \Theta) d \Theta \\
& \mathcal{J}_{n}^{2}=\frac{2}{\pi} \int_{0}^{\pi} \psi(\Theta) \cos (n \Theta) d \Theta=\frac{2}{\pi} \int_{0}^{\pi} \frac{\sin ^{2} \Theta \Theta}{\left(1+2 b \cos \Theta+b^{2}\right)^{2}} \cos (n \Theta) d \Theta
\end{aligned}
$$

We propose to compute the following integral using the residues theorem, where $a \in(0,1)$ :

$$
\mathcal{J}=\int_{0}^{\pi} \frac{\sin ^{2} \Theta}{\left(1-2 a \cos \Theta+a^{2}\right)^{2}} d \theta=\frac{1}{2} \int_{0}^{\pi} \frac{1}{\left(1-2 a \cos \Theta+a^{2}\right)^{2}} d \theta-\frac{1}{2} \int_{0}^{\pi} \frac{\cos 2 \Theta}{\left(1-2 a \cos \Theta+a^{2}\right)^{2}} d \Theta
$$

to find the result of the above integral which is composed by two integrals that can be written in the general form:

$$
\mathcal{J}=\int_{0}^{\pi} \frac{\cos k \Theta}{\left(1-2 a \cos \Theta+a^{2}\right)^{2}} d \Theta
$$

We use the variable change: $z=e^{i \Theta}$. Because $\Theta \in[0, \pi]$ the new domain will be the superior half plane of $|z|=1$. Thus the new form of $\mathcal{J}$ is:

$$
\mathcal{J}=\frac{1}{2 i} \int_{|z|=1} \frac{z^{2 k}+1}{z^{k-1}\left(a z^{2}-z\left(1+a^{2}\right)+a\right)^{2}} d z
$$


The singularities will be: $z=0 \in \partial D$ pole of $k-1$ degree and $z=a \in \partial D$ pole of second degree. Using the theorem of semi residues we finally obtain:

$$
\mathcal{J}=\frac{\pi a^{k}}{\left(1-a^{2}\right)^{3}}\left[k+1-(k-1) a^{2}\right]
$$

Replacing in (27) $k=0, k=2$ respectively, we obtain the result for $\mathcal{J}$ :

$$
\mathcal{J}=\frac{\pi}{2\left(1-a^{2}\right)}
$$

Under these conditions, for $a \rightarrow \frac{-b}{a^{2}}$ we obtain:

$$
\mathcal{J}_{0}^{1}=\frac{a^{4}}{a^{4}-b^{2}}
$$

and for $a \rightarrow-b$ we obtain:

$$
\mathcal{J}_{0}^{2}=\frac{1}{1-b^{2}}
$$

In analogous mode we compute the following integral:

$$
\begin{gathered}
\mathcal{J}_{n}(a)=\int_{0}^{\pi} \frac{\sin ^{2} \Theta}{\left(1-2 a \cos \Theta+a^{2}\right)^{2}} \cos (n \Theta) d \Theta=\frac{1}{2} \int_{0}^{\pi} \frac{1-\cos 2 \Theta}{\left(1-2 a \cos \Theta+a^{2}\right)^{2}} \cos (n \Theta) d \Theta= \\
=\frac{1}{2} \int_{0}^{\pi} \frac{\cos n \Theta}{\left(1-2 a \cos \Theta+a^{2}\right)^{2}} \cos (n \Theta) d \Theta-\frac{1}{2} \int_{0}^{\pi} \frac{\cos 2 \Theta \cos n \Theta}{\left(1-2 a \cos \Theta+a^{2}\right)^{2}} \cos (n \Theta) d \Theta \\
=\frac{1}{2} \int_{0}^{\pi} \frac{\cos n \Theta}{\left(1-2 a \cos \Theta+a^{2}\right)^{2}} \cos (n \Theta) d \Theta-\frac{1}{2} \int_{0}^{\pi} \frac{\cos (n+2) \Theta+\cos (n-2) \Theta}{2\left(1-2 a \cos \Theta+a^{2}\right)^{2}} \cos (n \Theta) d \Theta
\end{gathered}
$$

Using the (27) relation we have:

$$
\begin{gathered}
\mathcal{J}_{n}(a)=\frac{1}{2} \frac{\pi a^{n}}{\left(1-a^{2}\right)^{3}}\left(n+1-(n-1) a^{2}\right)-\frac{1}{4} \frac{\pi a^{n+2}}{\left(1-a^{2}\right)^{3}}\left(n+3-(n+1) a^{2}\right)-\frac{1}{4} \frac{\pi a^{n-2}}{\left(1-a^{2}\right)^{3}}\left(n-1-(n-3) a^{2}\right) \\
\mathcal{J}_{n}(a)=\frac{\pi a^{n-2}}{4\left(1-a^{2}\right)}\left(a^{2}(n+1)+1-n\right)
\end{gathered}
$$

Substituting in (28) $a \rightarrow-\frac{b}{a^{2}}$ we obtain:

$$
\mathcal{J}_{n}^{1}=\frac{\pi\left(-\frac{b}{a^{2}}\right)^{n-2}}{2\left(1-\left(-\frac{b}{a^{2}}\right)^{2}\right)}\left[\left(-\frac{b}{a^{2}}\right)^{2}(n+1)+1-n\right]
$$

and for $a \rightarrow-b$ we have:

$$
\mathrm{J}_{n}^{2}=\frac{\pi(-b)^{n-2}}{2\left(1-b^{2}\right)}\left(b^{2}(n+1)+1-n\right)
$$


In these conditions we can determine the coefficients from (25):

$$
\begin{gathered}
\alpha_{0}^{1}=-\frac{\Omega}{2 \mu} \frac{r_{2}^{2}\left(1-b^{2}\right)^{2}}{a^{4}} \mathcal{J}_{0}^{1}=-\frac{\Omega}{2 \mu} \frac{r_{2}^{2}\left(1-b^{2}\right)^{2}}{a^{4}-b^{2}} \\
\alpha_{0}^{2}=-\frac{\Omega}{2 \mu} r_{2}^{2}\left(1-b^{2}\right)^{2} \mathcal{J}_{0}^{2}=-\frac{\Omega}{2 \mu} r_{2}^{2}\left(1-b^{2}\right) \\
\alpha_{n}^{1}=-\frac{\Omega}{2 \mu} \frac{r_{2}^{2}\left(1-b^{2}\right)^{2}}{a^{4}} \mathcal{J}_{n}^{1}=-\frac{\Omega}{4 \mu} \frac{r_{2}^{2}\left(1-b^{2}\right)^{2}}{a^{4}} \frac{\pi\left(-\frac{b}{a^{2}}\right)^{n-2}}{\left(1-\left(-\frac{b}{a^{2}}\right)^{2}\right)}\left[\left(-\frac{b}{a^{2}}\right)^{2}(n+1)+1-n\right] \\
\alpha_{n}^{2}=-\frac{\Omega}{2 \mu} r_{2}^{2}\left(1-b^{2}\right)^{2} \mathcal{J}_{n}^{2}=-\frac{\Omega}{4 \mu} r_{2}^{2}\left(1-b^{2}\right) \pi(-b)^{n-2}\left(b^{2}(n+1)+1-n\right)
\end{gathered}
$$

With these computations we can find the Fourier coefficients (26):

$$
\begin{aligned}
& A=\frac{\Omega}{4 \mu \ln a} r_{2}^{2}\left(1-b^{2}\right) \frac{1-a^{4}}{a^{4}-b^{2}} \\
& B=-\frac{\Omega}{4 \mu} r_{2}^{2}\left(1-b^{2}\right) \frac{1-2 b^{2}+a^{4}}{a^{4}-b^{2}} \\
& a_{n}=\frac{1}{a^{2 n}-a^{-2 n}}\left\{\begin{array}{l}
-a^{n} \frac{\Omega}{4 \mu} r_{2}^{2}\left(1-b^{2}\right) \pi(-b)^{n-2}\left(b^{2}(n+1)+1-n\right)+ \\
+a^{-n} \frac{\Omega}{4 \mu} \frac{r_{2}^{2}\left(1-b^{2}\right)^{2}}{a^{4}} \frac{\pi\left(-\frac{b}{a^{2}}\right)^{n-2}}{\left(1-\left(-\frac{b}{a^{2}}\right)^{2}\right)}\left[\left(-\frac{b}{a^{2}}\right)^{2}(n+1)+1-n\right]
\end{array}\right\} \\
& b_{n}=\frac{1}{a^{2 n}-a^{-2 n}}\left\{\begin{array}{l}
a^{-n} \frac{\Omega}{4 \mu} r_{2}^{2}\left(1-b^{2}\right) \pi(-b)^{n-2}\left(b^{2}(n+1)+1-n\right)- \\
-a^{n} \frac{\Omega}{4 \mu} \frac{r_{2}^{2}\left(1-b^{2}\right)^{2}}{a^{4}} \frac{\pi\left(-\frac{b}{a^{2}}\right)^{n-2}}{\left(1-\left(-\frac{b}{a^{2}}\right)^{2}\right)}\left[\left(-\frac{b}{a^{2}}\right)^{2}(n+1)+1-n\right]
\end{array}\right\}
\end{aligned}
$$

\section{Numerical analysis}

In the following graphics (see fig. 3), the dependence of $W=W(R, \Theta)$ is represented using Maple software. It is observed that the solution is a stable one, and that there are no perturbations. 

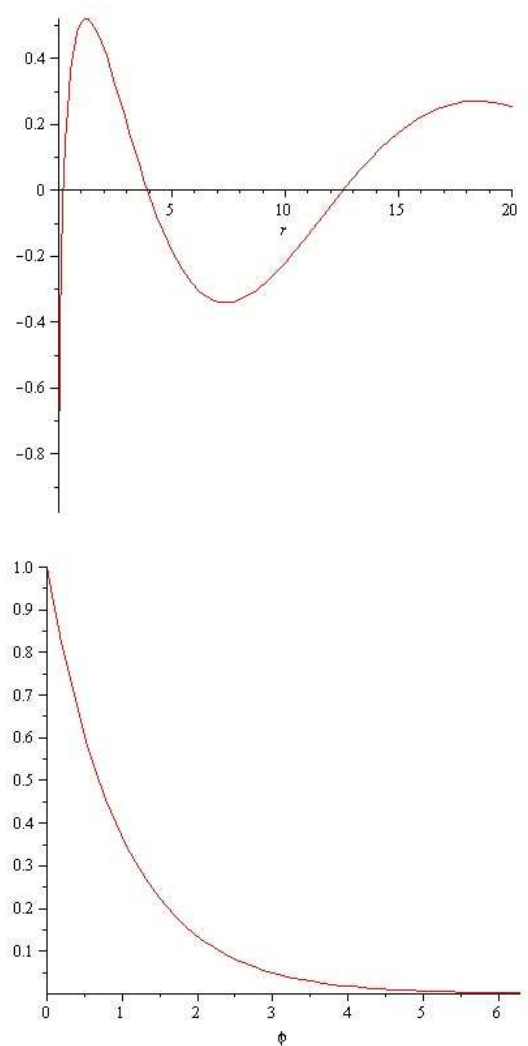

Figure 3: (a) Dependence of $\mathrm{W}$ by radius (b) Dependence of $\mathrm{W}$ by radius angle

\section{Acknowledgement}

This paper is supported by the Sectoral Operational Programme Human Resources Development (SOP HRD), financed from the European Social Fund and by the Romanian Government under the project number POSDRU/159/1.5/S/134378. 


\section{References}

[1] L. C. Berselli, F. Guerra, B. Mazzolai, E. Sinibaldi, Pulsatile Viscous Flows in Elliptical Vessels and Annuli: Solution to the Inverse Problem, with Application to Blood and Cerebrospinal Fluid Flow, SIAM J. Appl. Math., Vol. 74, number 1 (2012), 40-59

[2] P. Bhuanantanondh, P., Rheology of synovial fluid with and without Viscosupplements in patients with osteoarthritis: A pilot study, Biomed Eng Lett., 1(2011),213-219

[3] R. B. Bird, W. E. Stewart, E. N. Lig, Transport Phenomena, Wiley, New York, (1960).

[4] W. M. Brondani, Numerical study of a PTT visco elastic fluid flow through a concentric annular, Proceedings of COBEM (Brasillia), (2007).

[5] J. Enderle, J. Bronzino, Introduction to Biomedical Engineering, Academic Press, (2011)

[6] O. Florea, I-C. Rosca, A novel approach of the Stokes' second problem for the synovial fluid in knee osteoarthrosis, Journal of Osteoarthritis and cartilage Vol. 22 Supp (2014), S109-S110

[7] A.G Fredrickenson, R.B. Bird, Non Newtonian flow in annuli, Ind. Eng. Chem, vol. 50 (1958), 347-352

[8] R.W Hanks, K.M Larsen, The flow of power law non Newtonian fluid in concentric annuli, Ind. Eng. Chem. Fundam, vol. 18 (1979), pp 33-35

[9] L. Kopito, S. R. Schuster, H. Kosasky, Eccentric viscometer for testing biological and other fluids, patent number US3979945 A, (1976)

[10] X. Lin, B. Zhao and Z. Du, A third-order multi-point boundary value problem at resonance with one three dimensional kernel space, Carpathian Journal of Mathematics, Vol. 30 (2014), No. 1, 93-100

[11] M. Marin, O. Florea, On temporal behavior of solutions in Thermoelasticity of porous micropolar bodies, An. Sti. Univ. Ovidius Constanta, Vol. 22, issue 1,(2014), 169-188

[12] M. Marin, G. Stan, Weak solutions in Elasticity of dipolar bodies with stretch, Carpathian Journal Of Mathematics, Vol. 29, number 1, (2013), $33-40$ 
[13] M. Marin, Lagrange identity method in thermoelasticity of bodies with microstructure, International Journal of Engineering Science, vol. 32, issue 8, (1994), 1229-1240.

[14] M. Marin, Some estimates on vibrations in thermoelasticity of dipolar bodies, Journal of Vibration and Control, vol. 16 issue 1, (2010), 3347.

[15] F. T. Pinho, P. .J. Oliveira, Axial annular flow of a nonlinear viscoelastic fluid - an analytical solution, Journal Of Non-Newtonian Fluid Mechanics, 93 (2000), 325-337

[16] K. Sharma, M. Marin, Reflection and transmission of waves from imperfect boundary between two heat conducting micropolar thermoelastic solids, An. Sti. Univ. Ovidius Constanta, Vol. 22, issue 2,(2014), 151-175

[17] G. Zhi-rong, Y. Zong-yi, The eccentric effect of a coaxial viscometer, Applied Mathematics and Mechanics, pp. 359-367, 1995

[18] A. Wachs, Numerical Simulation of steady Bingham flow through an eccentric annular cross section by distributed Lagrange multipliers / fictitious domain and augmented Lagrangian methods, Non New Fluid Mech, vol. 142 (2007), pp183-198

Olivia FLOREA,

Department of Mathematics and Computer Science,

Transilvania University of Braşov,

Bdul Iuliu Maniu 50, Braşov, Romania.

Email: olivia.florea@unitbv.ro 